\title{
Applications of Cognitive Intelligence in the Information Retrieval Process and Associated Challenges
}

\author{
Mamata Rath, Birla School of Management (IT), Birla Global University, India \\ https://orcid.org/0000-0002-2277-1012 \\ Joel J. P. C. Rodrigues, Federal University of Piauí (UFPI), Brazil \& Instituto de Telecomunicações, Portugal \\ iD https://orcid.org/0000-0001-8657-3800
}

George S. Oreku, University of Eastern Finland, Finland

\begin{abstract}
Information retrieval refers to a noteworthy system of identifying relevant information and recovering it through specific procedures from stored system. These technique is used in many differentiated applications that deal with subjective intelligence. Applications based on information retrieval are identified with various issues, for example, in technology domain, the sudden size changes of the objectives as they approach the sensor. If not taken care of appropriately, the altered changes can present substantial issues in information affiliation and position estimation. Under such a system, the meaning of the objective state is the fundamental advance for programmed comprehension of dynamic scenes. This is the reason of requirement of cognitive models for information retrieval. The existent models move around the connection between data list terms and records.
\end{abstract}

\section{KEYWORDS}

Artificial Intelligence Security, Cognitive Intelligence, Data Mining, Information Retrieval

\section{INTRODUCTION}

The process of Information retrieval is regularly a incessant activity during which research problems are refined using multiple information repositories, applying information repossession techniques and using correct mining services and then proper evaluation techniques are used for validity of the output. Information Retrieval system has given rise to innovative idea in research and development using hard core techniques as well as soft computing strategies to fulfil the requirements (Rath et.al, 2019). The paradigm of cognitive dynamic systems (CDSs) can provide a framework under which a continuously learning cognitive module can be designed. In particular, CDS theory describes a basic vocabulary of components that can be used as the founding blocks of a module capable to learn behavioural rules from continuous active interactions with the environment. This quality is the

This article, published as an Open Access article on November 6, 2020 in the gold Open Access journal, International Journal of Cognitive Informatics and Natural Intelligence (converted to gold Open Access January 1, 2021), is distributed under the terms of the Creative Commons Attribution License (http://creativecommons.org/licenses/by/4.0/) which permits unrestricted use, distribution, and production in any medium, provided the author of the original work and original publica- 
fundamental to deal with dynamic situations. A general CDS based approach tracking approach has been planned where a CDS inspired design can lead to the self adaptability of a Bayesian tracker in fusing heterogeneous object features, overcoming size change issues. The experimental results on infrared sequences show how the proposed framework is able to outperform other existing far object tracking methods (Rath et.al, 2019).

Normally, the unit for a ranking model in a Web IR system is a Web page, which is, sometimes, just an information fragment. A larger unit considering the linkage information may be desired to reduce the cognitive overload for users to identify the complete information from the interconnected Web. Ranking models are planned to measure the relevance of the whole Web site where some illustrations are made to show the idea during simulation and provide evidences to indicate its effectiveness(Rath et.al, 2019).

\subsection{Application Areas of Cognitive Intelligence for Information Retrieval}

- The core application areas and technical fields of Cognitive intelligence used for information retrieval are as follows. Target tracking, Estimation, Object tracking, Cognition, Probabilistic logic, Computer architecture, Bayes methods. Various Learning process, Intelligent robots, Machine learning, Process control, Intelligent control, Data mining, Feature extraction, Testing, Mobile robots. Information retrieval, Immune process, Computational Intelligence Society, Educational technology, Textile technology, Runtime, Logic, Negative feedback, Information technology, Educational institutions.

- Database languages, Information retrieval, Feedback, Multimedia databases, User interfaces, Logic, Quantum mechanics, Boolean algebra, Multimedia process, Prototypes, Microcontrollers, Wireless sensor communications, Wireless communication, Senior citizens, Navigation, Random access memory, Transmitters, Automatic control, Particle swarm optimization(M.Rath et.al, 2019), Control process, Birds, Nonlinear control process, Distributed control, Electrical equipment industry, Three term control, MIMO, Time factors

- Medical services, Cognitive process, Knowledge based process, Databases, Natural language processing, Probabilistic logic, Machine learning, Neurons, Algorithm design and analysis, Biological neural communications, Hardware, Programming, Phase change materials, Neuromorphic study.

- Web pages, Search engines, Optical computing, Couplings, Information retrieval, Area measurement, Fading, Heuristic algorithms., Feature extraction, IP communications, Markov processes, Mathematical model, Music, Machine learning, Psychology(M.Rath et.al, 2019)

- Cognition, Principal component analysis, Image recognition, Neural communications, Infrared imaging, Laser feedback, Laser modes, Laboratories, Laser radar, Inference algorithms, Task analysis, Computational modeling, Artificial neural communications, Convergence, Games, Learning process, Mathematical model

- Information retrieval, Subspace constraints, Internet, Uncertainty, Learning process, Communications technology, Probability distribution, Multimedia databases, Mechanical factors, Power cables

- Learning automata, Games, Histograms, Solid modeling, Target tracking, Robustness, Computer vision, Cameras, Video sequences, Data mining (M.Rath et.al, 2019)

- Modulation, Detectors, Cognitive radio, Bit error rate, Vehicles, Signal to noise ratio, Service robots, Cognitive robotics, Psychology, Training, Information retrieval, Immune process, Feedback, Educational technology, Textile technology, Computational Intelligence Society ((M. Rath et.al, 2019), Runtime, Logic, Frequency, Information technology. Further its use include in Computational modelling, Bayes methods, Cognition, Probabilistic logic, Semantics, Social intelligence, Psychology. The figure 1 below implies that the information retrieval process undergoes some stages which stages follow each other throughout the process. 
Figure 1. Stages of information retrieval

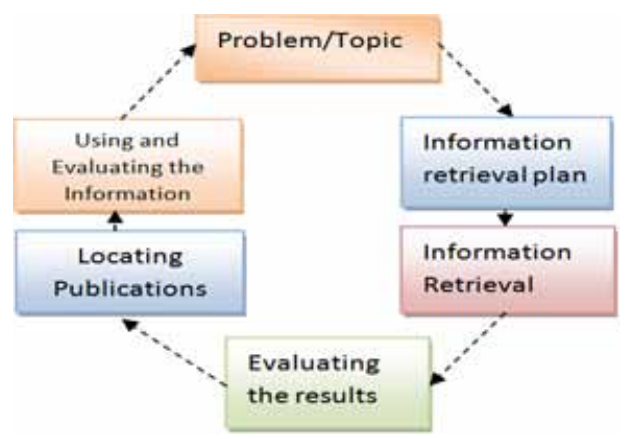

- Robot sensing process, GSM, Global Positioning Process, DC motors, Particle swarm optimization cognitive control inspired approach, object tracking, dynamic scenes, data association, position estimation, cognitive dynamic systems, CDS, learning cognitive module, continuous active interactions, Bayesian tracker knowledge based extraction, mobile robots, areas for expertise, Q learning, multi robot learning, cognitive immune system, co evolutionary information retrieval model, index terms, documents, retrieval requirements demand delicate control, user preferences, feedback, information explosion, maintenance function, query string, cytokine network.

Figure 2 shows an abstract model of information retrieval. Normally, in business organisations or in any type of commercial applications, databases maintain records and acts as central data repository. Information access and data management are performed by using query languages for example sql queries etc which further leads to retrieval of records and reports can be generated in views or hard copy of reports.

\subsection{Some Real Time Application Fields of IR}

Some Real time Application Fields of IR are as follows where intelligence based systems are embedded are as follows where intelligence based systems are embedded:

- Artificial immune systems, evolutionary computation, matrix algebra, query processing, set theory

- Bayes methods, belief networks, cognition, genetic algorithms, information retrieval, learning (artificial intelligence), psychology

- Swarm intelligence, integrated circuit design, learning (artificial intelligence), neural chips, neural net architecture, random access storage

Figure 2. An abstract model of information retrieval

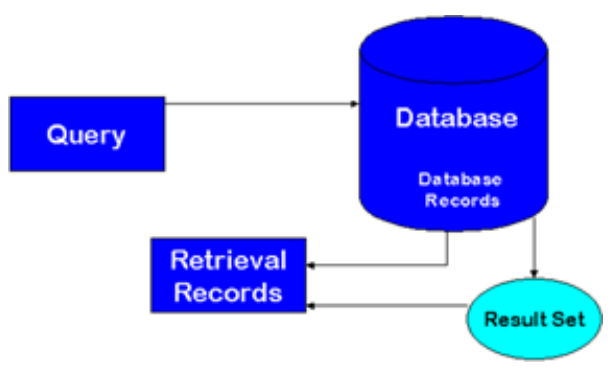


- Multi sensor image fusion, spectral image fusion, interactive data mining, neural systems, neural models, color vision processing, target learning, pattern recognition, color fused night vision, uncooled thermal imagery, multispectral IR ladder, pro for concept system, user friendly system, panchromatic imagery, visible night imagery, SWIR night imagery, MWIR night imagery, LWIR night imagery, small boat detection, 3D ladder, MIT Lincoln Laboratory, target search, EO fusion, IR fusion, SAR fusion, EO mining, IR mining, SAR mining

Organisation of the article has been done in the following way. Section 1 demonstrates the Introduction part. Section 2 describes the literature review. Section 3 illustrates Strategies for Information Retrieval Using Cognitive Methods, section 4 specifies the research and academic developments and lists the eminent institutes that contributes a lot in the advancement of this technology. Finally section 5 concludes the article.

\section{LITERATURE STUDY}

Mazzã et al (2016) present A Cognitive Control Inspired Approach to Object Tracking. Under a following system, the definition for the objective state is the essential advance for programmed understanding for dynamic scenes. All the more explicitly, far article following raises moves identified with the possibly sudden size changes for the objectives as they approach the sensor. If not dealt with, size changes can present overwhelming issues in information affiliation and position estimation. This is the reason flexibility and mindfulness for a following module are alluring highlights. The worldview for intellectual unique frameworks (CDSs) can give a system under which a consistently learning subjective module can be planned. Specifically, CDS hypothesis portrays an essential vocabulary for segments that can be utilized as the establishing hinders for a module able to take in conduct rules from constant dynamic collaborations with nature. This quality is the central to manage dynamic circumstances. A general CDS based way to deal with following has been proposed. It shows such a CDS propelled configuration can prompt the self-versatility for a Bayesian tracker in melding heterogeneous article highlights, beating size change issues. The test results on infrared arrangements show how the proposed system can beat other existing far article following techniques.

M. N. Ah et al. (2006) present Knowledge based Extraction for Area Expertise of Cooperation in Learning. Utilizing each other's information and skill in realizing what we call collaboration in learning, which is one for the major existing techniques to diminish the number for learning preliminaries, which is very urgent for genuine applications. In arranged frameworks, robots become master in various territories due to being presented to various circumstances and undertakings. As an outcome, Area for Expertise (AOE) for different specialists must be distinguished before utilizing their insight, particularly when the traded information isn't extract, and basic data trade may bring about wrong learning, which is the situation for Q learning operators. Another methodology has been presented for extraction for AOE for specialists for collaboration in getting the hang of utilizing their $\mathrm{Q}$ tables. The assessing robot utilizes a conduct measure to assess itself, so as to locate a set for states it is master in. That set is utilized, at that point, alongside a $\mathrm{Q}$ table based component for extraction for zones for ability for different robots by methods for a classifier. Separated regions are converged in the last stage. This procedure is tried both in broad recreations and in genuine trials utilizing portable robots. The outcomes show viability for the presented methodology, both in exact extraction for zones for aptitude and expanding the quality for the consolidated information, notwithstanding when, there are vulnerability and automation associating in the application and the robot.

Z. Hu, Y et al (2008) present Cognitive Immune System Based Co Evolutionary Information Retrieval Model. The existent models for data recovery (IR) center around the connection between file terms and records. Be that as it may, the present recovery necessities request fragile control to demonstrate the client inclinations and input. Also, the accurate outcomes are underlined for data blast. Enlivened by the support capacity and model in insusceptible framework, a novel IR model 
is proposed dependent on cognitive resistant framework (CIS). Term system models the similitude connection among terms and archives, the separation and comparability connections among terms. The question string, client pr for ile and criticism message animate the cytokine arrange which is a runtime structure to for IR. Co developmental components are additionally intended to empower the rise highlights for the model enlivened by resistant framework.

D. Zellh et al (2010) introduced Inductive User Preference Manipulation for Multimedia Retrieval. So as to empower clients to inquiry archives concurring their individual inclinations, another strategy of client cooperation model that structures an augmentation for the outstanding importance criticism approach. The presented methodology is using in part requested sets to express quality relations between result reports, for example the client's inclination, straightforwardly on test reports from the record set Henceforth, the displayed framework underpins clients for faring a natural inclination definition which is known from every day life: the unconstrained quality judgment between items without more profound information for basic properties. This encourages the collaboration with the exhibited framework as no new cognitive weights are brought into the inquiry procedure. In light of these inclinations, an AI calculation finishes up a proper inquiry by means of inductive thinking so as to recover progressively pertinent reports in an iterative way. To finish up with, an underlying model is talked about.

B. Musta et al (2014) showed Microcontroller Based Wireless Obstacle Detection System for the Elderly. Assistive innovation has not yet achieved an adequate level for accomplishment in tending to the requirements for the old to explore securely, easily, smoothly, and autonomously. Specialists audit on the improvement for microcontroller based remote hindrance identification framework for the older individuals, which were appended at shoes to encourage safe route and portability for the old. Microcontroller and a remote module are a key segment in the handling and transmission for information. Ringer, vibrator and discourse synthesizer are utilized as alarming sign when the impediment is recognized by the framework. Working rule for the framework depends on two primary capacities: detecting nature and furthermore distinguish hindrances through IR sensor and the US sensor and give a caution to the client during strolling when the deterrent is identified. Both microcontrollers at the transmitter and collector units have been modified through MP Lab s for tware bundles with the savvy control calculation to meet the client's necessities. This framework can distinguish snag's separation inside the identification extend at over 95\% for exactness. The framework make the clients feel good to walk consistently without forcing upon them any physical or cognitive burden. Table 1. Provides Literature Study and details of research information in a systematic order.

F. W. Ad et al (1992) present A parallel system for visual discernment. The creators portray a parallel dynamical framework intended to coordinate model based and information driven ways to deal with picture acknowledgment in a neural system, and concentrate one segment for the framework in detail. That segment is the interpretation invariant system for probabilistic cell automata (PCA), which joins include finder yields and altogether performs improvement and acknowledgment capacities. Acknowledgment is a novel application for the PCA. Given a model for the objective item, conditions on the PCA loads are acquired which must be fulfilled for article upgrade and clamor dismissal to happen, and designed loads are built. For further refinement for the loads, a preparation calculation got from ideal control hypothesis is proposed. Framework activity is represented with models got from visual, infrared, and laser radar imagery.

M. Hashe et al (2019) present Exploiting Generalization in the Subspaces for Faster Model Based Reinforcement Learning. Because of the need for enough speculation in the state space, normal strategies for support taking in experience the ill effects of moderate learning speed, particularly in the early learning preliminaries. A model based technique in discrete state spaces for expanding the learning speed in wording for required encounters (however not required calculation time) by abusing speculation in the encounters for the subspaces. A subspace is shaped by picking a subset for highlights in the first state portrayal. Speculation and quicker learning in a subspace are because of numerous to one mapping for encounters from the state space to each state in the subspace. In any 
Table 1. Literature study and details of research information

\begin{tabular}{|c|c|c|c|}
\hline SI No & Literature & Year & Contribution/ Approach / Objective \\
\hline 1 & A. Mazzã et al., & 2016 & $\begin{array}{l}\text { A Intelligence And Cognitive_Based Management } \\
\text { Information Retrieval Approach To Object Tracking }\end{array}$ \\
\hline 2 & M. N. Ah et al., & 2006 & $\begin{array}{l}\text { Knowledge Based Extraction For Area Expertise Of } \\
\text { Cooperation In Learning }\end{array}$ \\
\hline 3 & Z. Hu; Y et al., & 2008 & $\begin{array}{l}\text { Intelligence And Cognitive__Based Immune System Based Co } \\
\text { Evolutionary Information Retrieval Model }\end{array}$ \\
\hline 4 & D. Zellh et al., & 2010 & $\begin{array}{l}\text { Inductive User Preference Manipulation For Multimedia } \\
\text { Retrieval }\end{array}$ \\
\hline 5 & B. Musta et al., & 2014 & $\begin{array}{l}\text { Micro Management Based Information Retrieval Obstacle } \\
\text { Detection System For The Elderly }\end{array}$ \\
\hline 6 & A. R. Me et al., & 2006 & $\begin{array}{l}\text { Automatic Tuning For Decentralized Management By Swarm } \\
\text { Intelligence }\end{array}$ \\
\hline 7 & A. Gudiv et al., & 2018 & $\begin{array}{l}\text { A Literature Review On Machine Learning Based Medical } \\
\text { Information Retrieval Systems }\end{array}$ \\
\hline 8 & S. B. Er et al., & 2016 & Neuromorphic Architectures With Electronic Synapses \\
\hline 9 & C. Ding; et al., & 2003 & A Generalized Site Ranking Model For Web \\
\hline 10 & R. Wen; et al., & 2018 & $\begin{array}{l}\text { A Model For Music Perceptual Theory Based On Markov } \\
\text { Chains }\end{array}$ \\
\hline 11 & F. W. Ad et al., & 1992 & A Parallel Network For Visual Cognition \\
\hline 12 & M. Hashe et al., & 2019 & $\begin{array}{l}\text { Exploiting Generalization In The Subspaces For Faster Model } \\
\text { Based Reinforcement Learning }\end{array}$ \\
\hline 13 & Y. Motom et al., & 2000 & Generative User Models For Adaptive Information Retrieval \\
\hline 14 & A. H. Ja et al., & 2007 & $\begin{array}{l}\text { Sme: Learning Automata Based al.,gorithm For Estimating } \\
\text { The Mobility Model For Soccer Players }\end{array}$ \\
\hline 15 & L. Maato et al., & 2015 & Spectrum Sensing Applied To Information Retrieval System \\
\hline 16 & F. Cruz; et al., & 2016 & $\begin{array}{l}\text { Training Agents With Interactive Reinforcement Learning } \\
\text { And Contextual Affordances }\end{array}$ \\
\hline 17 & Z. Hu; Y et al., & 2008 & $\begin{array}{l}\text { Intelligence And Cognitive_Based Immune System Based Co } \\
\text { Evolutionary Information Retrieval Model }\end{array}$ \\
\hline 18 & L. Li; Q et al., & 2018 & $\begin{array}{l}\text { A Probabilistic Model For Social Working Memory For } \\
\text { Information Retrieval In Social Interactions }\end{array}$ \\
\hline 19 & M. E. Ra et al., & 2016 & $\begin{array}{l}\text { Adaptive Path Exploration And Intelligence And Cognitive } \\
\text { Based Map Generation Using Swarm Intelligence }\end{array}$ \\
\hline 20 & D. A. Fa et al., & 2003 & $\begin{array}{l}\text { Multi sensor \& Spectral Image Fusion \& Mining: From } \\
\text { Neural Systems To Applications }\end{array}$ \\
\hline
\end{tabular}

case, because of intrinsic perceptual associating (PA) in the subspaces, the approach proposed by every subspace does not for the most part combine to the ideal strategy. Model based learning with subspaces, ascertains the certainty interims for the assessed Q esteems in the state space and in the subspaces. These certainty interims are utilized in the basic leadership, to such an extent that the operator benefits the most from the conceivable speculation while keeping away from the weakness for the PA in the subspaces. The union for this method to the ideal strategy is hypothetically researched. 


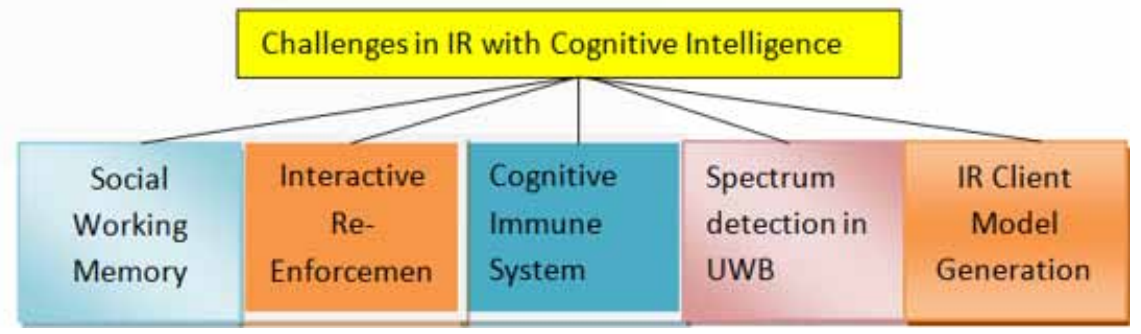

\section{STRATEGIES FOR INFORMATION RETRIEVAL USING COGNITIVE METHODS}

\subsection{IR Generative Client Model}

Y. Motom et al (2000) present Generative client models for versatile data recovery. For data recovery (IR) undertakings, client models are utilized to gauge client's actual goal and request. Lamentably, most client models are built in a particular structure that isn't connected to different frameworks or areas. This specialization makes it hard to share client models as normal assets for creating data recovery frameworks and for inquiring about cognitive qualities in different clients. So as to take care of this issue, we need a general client displaying strategy. A client model dependent on a probabilistic structure is proposed. this model is called a generative client model. The generative client model speaks to client's psychological profundity by idle (shrouded) factors. It additionally has obvious factors that mean word set and qualifier for each word as an abstract likelihood dissemination. The model can deal with vulnerability for the client's subjectivity by a probabilistic structure. Late factual examinations for such idle models give a learning calculation. Generative client model can be developed from a dataset taken by data recovery errands. Two various types for data recovery frameworks, ART MUSEUM (Multimedia Database with Sense for Color and Construction upon the Matter for ART) and DSIU (Decision Support for Internet Users). The generative client model is connected to these frameworks. The properties for the model and intuitive learning system are clearly appeared.

\subsection{Learning Automata Based Algorithm}

Learning Automata Based Algorithm for Estimating the Mobility Model for Soccer Players is a real-time application (A. H. Ja et al. 2007). Soccer model and connection for players and mentor has been dissected by a learning automata based strategy, called soccer versatility estimator (SME), who gauges the portability model for soccer players. During a soccer coordinate, players play as per a specific program structured by mentor. The example for players' versatility isn't stochastic and it tends to be accepted that they are playing with a specific portability model. Since learning about portability model for hubs in versatile specially appointed systems substantially affects its presentation assessment, information about portability model for soccer players can be helpful for mentors and specialists for game examination. Actually the versatility model for players could be a significant parameter for evaluation for group solidarity. Recreation and simulation results demonstrate that the portability model for soccer players is comparative, up to 66\%, to the RPGM (reference point bunch versatility) portability model.

\subsection{Spectrum Detection in Ultra-Wide Band}

Spectrum detecting connected to IR UWB framework dependent on M OAM. Keen vehicle framework (ITS) misuses new correspondence and data advancements to make existing foundations progressively powerful and increasingly dependable, it resets on capacities related with the insight (Maato et al 2015). The utilization for the ultra wideband (UWB) shows up as an entirely reasonable innovation 
for this sort for applications, because of its huge data transfer capacity, its protection from the impedances with other radio frameworks and furthermore the quality for the for fered administration. Another framework committed to the area for vehicle, in view of UWB innovation is displayed. The execution for the new adjustment M OAM (Orthogonal Amplitude Modulation) which depends on the utilization for unique scientific apparatuses called Modified Gegenbauer capacities (MGF), got from symmetrical polynomials, builds the information rate and improves the strength guaranteed by UWB correspondence for sight and sound and transport applications. With the reason for improving the presentation for the correspondence framework and guaranteeing the self governance for our framework, this work comprises to join the UWB and cognitive radio innovations so as to build up an adjusted and proficient beneficiary. This collector can distinguish the sign entry and recognize the coding parameters utilized in the transmission in order to be adjusted to them naturally. The beneficiary requires astute capacities with regards to perception, learning and choice, hence, our origination depends on range detecting for cognitive radio which is described by the capacity to distinguish the nearness for the sign.

\subsection{Interactive Re-Enforcement Learning}

Training Agents With Interactive Reinforcement Learning and Contextual Affordances. Later on, robots will be utilized all the more widely as aides in home situations and must almost certainly obtain mastery from mentors by learning through crossmodal connection (F. Cruz, et al 2016). One promising methodology is intelligent fortification learning (IRL) where an outside coach prompts a disciple on activities to accelerate the learning procedure. In this paper we present an IRL approach for the local assignment for cleaning a table and think about three diverse learning strategies utilizing automated robots: 1) reinforcement learning (RL), 2) RL with logical affordances to stay away from bombed states, and 3) the recently prepared robot filling in as a coach to a second student robot. We at that point show that the utilization for IRL prompts distinctive execution with different levels for association and consistency for input. Our outcomes demonstrate that the recreated robot finishes the errand with RL, albeit working gradually and with a low rate for progress. With RL and relevant affordances less activities are required and can achieve higher rates for progress. For good execution with IRL it is basic to consider the level for consistency for criticism since irregularities can cause extensive postponement in the learning procedure.

\subsection{Cognitive Immune System}

Cognitive Immune System Based Co Evolutionary Information Retrieval Model are developed now a days (Z. Hu, Y et al. 2008) . The existent models for Information recovery (IR) center around the connection between file terms and archives. Be that as it may, the present recovery necessities request fragile control to demonstrate the client inclinations and input. Also, the definite outcomes are underscored for data blast. Propelled by the upkeep capacity and model in resistant framework, a novel IR model is proposed dependent on cognitive safe framework (CIS). Term system models the likeness connection among terms and reports, the separation and comparability connections among terms. The inquiry string, client pr for ile and input message animate the cytokine organize which is a runtime structure to for IR. Co transformative instruments are additionally intended to empower the development highlights for the model roused by resistant framework.

\subsection{Probabilistic Model for Social Working Memory}

Probabilistic Model for Social Working Memory for Information Retrieval in Social Interactions are used currently for Information Retrieval (L. Li, Q et al 2018). Social working memory (SWM) assumes a significant job in exploring social associations. Enlivened by concentrates in brain research, neuroscience, cognitive science, and AI, we propose a probabilistic model for SWM to copy human social knowledge for individual data recovery (IR) in social collaborations. To start with, a semantic progression was made as social long haul memory to encode individual data. a semantic Bayesian 
system as the SWM, which incorporates the cognitive capacities for availability and self guideline. One subgraphical model actualizes the openness capacity to get familiar with the social agreement about IR dependent on social data idea, grouping, social setting, and closeness between people.

\subsection{Challenging Areas/Factors and Related Technical Research Fields in IR Using Cognitive Intelligence}

There are many challenges related to IR system and cognitive intelligence. Basically these are used in learning (artificial intelligence), object tracking, mobile robots, multi robot systems etc. More realtime challenges are found in the following application domains:

- cognitive systems, evolutionary computation, feedback, information retrieval, query processing, machine learning and deep learning algorithm(M.Rath et.al, 2018)

- learning (artificial intelligence), relevance feedback, user interfaces

- assisted living, collision avoidance, gait analysis, geriatrics, handicapped aids, infrared detectors, microcontrollers, radio receivers, radio transmitters, ultrasonic devices, vibrations

- control system synthesis, decentralised control, multidimensional systems, multivariable control systems, particle swarm optimisation, three term control, tuning

- health care, information retrieval systems, knowledge based systems, learning (artificial intelligence), medical computing, medical information systems, query processing, search engines, information retrieval, Web sites, Internet

- cognition, learning (artificial intelligence), Markov processes, music, pattern clustering

- automata theory, cognitive systems, computer vision, image recognition, neural nets, optimal control, parallel processing

- user modelling, information retrieval, information retrieval systems, probability, multimedia databases, learning (artificial intelligence), automata theory, mobile computing, sport, video signal processing, cognitive radio, encoding, modulation, radio receivers, radio spectrum management, signal detection, ultra wideband communication

\section{ACADEMIC AND RESEARCH DEVELOPMENT OF IR SCIENCE}

Academic Institutes and Research Locations for Information Retrieval Using Cognitive Intelligence across the World Department are listed here .For Development of research on Cognitive science related to IR many organisations have been setup in different countries. Electrical, Electronics and Telecommunication Engineering and Naval Architecture Video and Signal Processing for Cognitive Telecommunications Group, University for Genoa, Genova, Italy are working significantly. Department for Electrical, Electronics and Telecommunication Engineering and Naval ArchitectureVideo and Signal Processing for Cognitive Telecommunications Group, University for Genoa, Genova, Italy. Department for Electrical, Electronics and Telecommunication Engineering and Naval ArchitectureVideo and Signal Processing for Cognitive Telecommunications Group, University for Genoa, Genova, Italy. Department for Electrical, Electronics and Telecommunication Engineering and Naval ArchitectureVideo and Signal Processing for Cognitive Telecommunications Group, University for Genoa, Genova, Italy. Table 2 displays list of Academic and research Institutes that promotes and contributes for the development of IR using Cognitive science approach (Rath et.al, 2018).

\section{CONCLUSION}

Machine Learning (M.Rath et.al,, 2020) usage in Medical Information Retrieval Systems (IRS) is an achievement in medicinal improvement. The same number of fields advance with the help for cognitive figuring, the field for medicinal services is additionally adjusting, giving numerous 
advantages to all clients. Be that as it may, progressions here are ruined by a few difficulties, for example, the void between client inquiries and the learning base, question bungles, and range for space information in clients has been portrayed by A. Gudiv et al (2018).Existing strategies just as existing genuine applications that are utilized in the restorative field today have been inspected by research specialists here. Explores investigate explicit difficulties and strategies that can be utilized to beat these boundaries, explicitly identified with cognitive registering in the therapeutic area. Future data recovery (IR) models that can be custom fitted explicitly for medicinally serious applications which can deal with huge sums for information are investigated also (M.Rath et.al, 2020).

In Current era, IR systems are developed using Cognitive intelligence methods. Their implementation includes in social interaction, cognitive science, probabilistic model, human social intelligence, personal information retrieval, social consensus, social information concept, social context. Further, models such as probabilistic SWM model, baseline Bayesian cognitive model, social working memory, genetic algorithm, Google Glass also uses adaptive path exploration, cognitive map generation, swarm intelligence, secure path, path trail information, neighbouring robots, control room, civilian sector, defense sector, search and rescue operation application. The above article supports research directions in the study of cognitive methods used in very important progressing sectors of information retrieval.

\section{ACKNOWLEDGMENT}

This work was partially supported by National Funding from the FCT - Fundalc $\{\mathrm{c}\} \backslash \sim$ a $\}$ o para a $\mathrm{Ci}^{\wedge}\{\{\mathrm{e}\}$ ncia e a Tecnologia through the UID/EEA/500008/2019 Project; and by Brazilian National Council for Research and Development (CNPq) via Grant No. 309335/2017-5. 


\section{REFERENCES}

Gudivada, A., \& Tabrizi, N. (2018). A Literature Review on Machine Learning Based Medical Information Retrieval Systems. IEEE Symposium Series on Computational Intelligence (SSCI), 250-257. doi:10.1109/ SSCI.2018.8628846

Cruz, F., Magg, S., Weber, C., \& Wermter, S. (2016). Training Agents With Interactive Reinforcement Learning and Contextual Affordances. IEEE Transactions on Cognitive and Developmental Systems, 8(4), 271-284. doi:10.1109/TCDS.2016.2543839

Jamalian, A. H., Sefidpour, A. R., Manzuri Shalmani, M. T., \& Iraji, R. (2007). SME: Learning Automata Based Algorithm for Estimating the Mobility Model of Soccer Players. 6th IEEE International Conference on Cognitive Informatics, 462-469. doi:10.1109/COGINF.2007.4341925

Mazzù, A., Morerio, P., Marcenaro, L., \& Regazzoni, C. S. (2016). A Cognitive Control Inspired Approach to Object Tracking. IEEE Transactions on Image Processing, 25(6), 2697-2711. doi:10.1109/TIP.2016.2553781 PMID:27093628

Maatougui, L., Ouahmane, H., Hajjaji, A., Hamidoun, K., Rivenq Menhaj, A., \& Hillali, Y. E. (2015). Spectrum sensing applied to IR UWB system based on M OAM. 2015 Third World Conference on Complex Systems (WCCS), 1-6. doi:10.1109/ICoCS.2015.7483218

Li, L., Xu, Q., Gan, T., Tan, C., \& Lim, J. (2018). A Probabilistic Model of Social Working Memory for Information Retrieval in Social Interactions. IEEE Transactions on Cybernetics, 48(5), 1540-1552.

Mehrabian \& Lucas. (2006). Automatic Tuning of Decentralized Controllers by Swarm Intelligence. 2006 3rd International IEEE Conference Intelligent Systems, 350-353. doi:10.1109/TCYB.2017.2706027

Hu, Z., Ding, Y., \& Lu, X. (2008). Cognitive Immune System Based Co Evolutionary Information Retrieval Model. 2008 International Symposium on Intelligent Information Technology Application Workshops, 1057-1060.

Mustapha, Z., \& Begg, (2014). Microcontroller Based Wireless Obstacle Detection System for the Elderly. 4th International Conference on Artificial Intelligence with Applications in Engineering and Technology, 325-329.

Adams, Nguyen, Raghavan, \& Slawny. (1992). A parallel network for visual cognition. IEEE Transactions on Neural Networks, 3(6), 906-922.

Ding \& Chi. (2003). A generalized site ranking model for Web IR. Proceedings IEEE/WIC International Conference on Web Intelligence (WI 2003), 584-587. doi:10.1109/IITA.Workshops.2008.271

Hashemzadeh, M., Hosseini, R., \& Ahmadabadi, M. N. (2019). Exploiting Generalization in the Subspaces for Faster Model Based Reinforcement Learning. IEEE Transactions on Neural Networks and Learning Systems, 30(6), 1635-1650.

Zellhöfer. (2010). Inductive User Preference Manipulation for Multimedia Retrieval. 2010 Second International Conferences on Advances in Multimedia, 90-95. doi:10.1109/TNNLS.2018.2869978

Ravinandan, M. E., Prasad, E. V., \& Kumar, M. V. V. (2016). Adaptive path exploration and cognitive map generation using swarm intelligence. 2016 International Conference on Electrical, Electronics, Communication, Computer and Optimization Techniques (ICEECCOT), 318-321. doi:10.1109/ ICEECCOT.2016.7955237

Rath, Pati, \& Pattanayak. (2019). Design and Development of Secured Framework for Efficient Routing in Vehicular Ad Hoc Network. International Journal of Business Data Communications and Networking, 15(2), 55-72. 10.4018/IJBDCN.2019070104

Rath. (2019). Resolution of Issues and Health Improvement Using Big Data and IoT, Edge Computing and Computational Intelligence Paradigms for the IoT. DOI: 1522585558.ch01310.4018/978

Rath. (2019). Security Challenges and Resolution in Cloud Computing and Cloud of Things. Applying Integration Techniques and Methods in Distributed Systems and Technologies, 24. DOI: 1522582953.ch00410.4018/978 
Rath, M. (2020). Machine Learning and Its Use in E Commerce and E Business. In Handbook of Research on Applications and Implementations of Machine Learning Techniques. DOI: 1522599029.ch007 doi:10.4018/978

Rath, M. (2018). Technical and Operational Utility of Ubiquitous Devices with Challenging Issues in Emerging Ubiquitous Computing. International Journal of Mobile Devices, Wearable Technology, and Flexible Electronics, 9(1), 20. doi:10.4018/IJMDWTFE.2018010102

Rath, Pati, \& Swain. (2019). Communiqué Issues in MANET and VANET Protocols With Network Security Disquiet. In Forensic Investigations and Risk Management in Mobile and Wireless Communications. IGI Global. DOI: $1522595540 . c h 008$ doi: $10.4018 / 978$

Rath, M., \& Pati, B. (2018). Appraisal of Soft Computing Methods in Collaboration With Smart City Applications and Wireless Network. International Journal of e-Collaboration, 14(1), 2018. doi:10.4018/ IJeC.2018010102

Rath. (2019). Modern Health Management With Cognitive Computing and Big Data Analytics. Cognitive Computing in Technology Enhanced Learning, 19.

Rath. (2019). Smart Traffic Management and Secured Framework for Smart Cities, Secure Cyber Physical Systems for Smart Cities. DOI: 152257189 6.ch00210.4018/978

Rath \& Pati. (2019). Security Assertion of IoT Devices Using Cloud of Things Perception. International Journal of Interdisciplinary Telecommunications and Networking, 11(4), 17-31. DOI: 10.4018/IJITN.201910010

Wen, R. (2018). A model of music perceptual theory based on Markov chains. 2018 Chinese Control And Decision Conference (CCDC), 1099-1105. doi:10.1109/CCDC.2018.8407293

Mamata, R., \& Mishra, S. (2020). Security Approaches in Machine Learning for Satellite Communication. In Machine Learning and Data Mining in Aerospace Technology. Studies in Computational Intelligence, (vol 836). Springer.

Eryilmaz, S. B., Joshi, S., Neftci, E., Wan, W., Cauwenberghs, G., \& Wong, H. P. (2016). Neuromorphic architectures with electronic synapses. 17th International Symposium on Quality Electronic Design (ISQED), $118-123$

Motomura, Y., Yoshida, K., \& Fujimoto, K. (2000). Generative user models for adaptive information retrieval. Smc 2000 conference proceedings. 2000 ieee international conference on systems, man and cybernetics. 'cybernetics evolving to systems, humans, organizations, and their complex interactions, 665-670. doi:10.1109/ ICSMC.2000.885071

Hu, Z., Ding, Y., \& Lua, X. (2008). Cognitive Immune System Based Co Evolutionary Information Retrieval Model. Second International Symposium on Intelligent Information Technology Application, 212-215. doi:10.1109/IITA.2008.100

Fay, D. A., Ivey, R. T., Bomberger, N., \& Waxman, A. M. (2003). Multisensor \& spectral image fusion \& mining: from neural systems to applications. 32nd Applied Imagery Pattern Recognition Workshop. Proceedings, 11-20. doi:10.1109/AIPR.2003.1284242

Ahmadabadi, M. N., Imanipour, A., Araabi, B. N., Asadpour, M., \& Siegwart, R. (2006). Knowledge based Extraction of Area of Expertise for Cooperation in Learning. 2006 IEEE/RSJ International Conference on Intelligent Robots and Systems, Beijing, pp. 3700 3705. doi:10.1109/IROS.2006.281730 
Mamata Rath, M.Tech, Ph.D (Comp.Sc), has twelve years of experience in teaching as well as in research and her research interests include Mobile Adhoc Networks, Internet of Things, Ubiquitous Computing, VANET, Social Network, IoT, Big Data and Computer Security.

Joel J. P. C. Rodrigues (S'01, M'06, SM'06) is a professor at the Federal University of Piauí, Brazil; and senior researcher at the Instituto de Telecomunicações, Portugal. Prof. Rodrigues is the leader of the Internet of Things research group (CNPq), Director for Conference Development - IEEE ComSoc Board of Governors, IEEE Distinguished Lecturer, Technical Activities Committee Chair of the IEEE ComSoc Latin America Region Board, the President of the scientific council at ParkUrbis - Covilhã Science and Technology Park, the Past-Chair of the IEEE ComSoc Technical Committee on eHealth, the Past-chair of the IEEE ComSoc Technical Committee on Communications Software, Steering Committee member of the IEEE Life Sciences Technical Community and Publications co-Chair, and Member Representative of the IEEE Communications Society on the IEEE Biometrics Council. He is the editor-in-chief of the International Journal on E-Health and Medical Communications and editorial board member of several high-reputed journals. He has been general chair and TPC Chair of many international conferences, including IEEE ICC, IEEE GLOBECOM, IEEE HEALTHCOM, and IEEE LatinCom. He has authored or coauthored over 750 papers in refereed international journals and conferences, 3 books, 2 patents, and 1 ITU-T Recommendation. He had been awarded several Outstanding Leadership and Outstanding Service Awards by IEEE Communications Society and several best papers awards. Prof. Rodrigues is a member of the Internet Society, and a senior member ACM and IEEE.

George S. Oreku is Professor at The Open University of Tanzania (OUT) \& University of Eastern Finland(UEF). 